\title{
PREFERRED ORIENTATION OF MnS INCLUSIONS IN ROLLED STEEL
}

\author{
A. JOHN MARDINLY, LAWRENCE H. VAN VLACK AND \\ WILLIAM F. HOSFORD \\ Dept. of Materials Science and Engineering, The University of Michigan \\ Ann Arbor, Mich 48109-2136, USA
}

\begin{abstract}
The preferred orientation of $\mathrm{MnS}$ inclusions in hot-rolled steel was studied by electron diffraction. Specimens of resulfurized steel were examined after casting and after hot rolling to true strains of $0.5,1.0$ and 1.5. After rolling, the orientations of the inclusions tended toward an ideal $\langle 100\rangle\{001\}$. Only a moderate correlation between the final inclusion shape and orientation was found.
\end{abstract}

KEY WORDS: Steel, hot rolling, MnS inclusions, selected area diffraction, preferred orientation.

\section{INTRODUCTION}

This paper addresses the question of whether the $\mathrm{MnS}$ inclusions in rolled steel have a preferred orientation. Details of the study are reported elsewhere Mardinly. As steel is hot rolled, MnS inclusions deform as well as the steel itself; the relative amount of deformation of the inclusions and the steel varying with temperature. The primary slip system for MnS is $\langle 110\rangle\{110\}$. Chao, Thomassen, and Van Vlack. Chao and Van Vlack studied the deformation of single crystal MnS inclusions in low-carbon steel during uniaxial compression and found that inclusions oriented with $<100\rangle$ parallel to the compression axis deformed more than other inclusions of other orientations because the $\langle 110\rangle\{110\}$ slip systems were more favorably oriented.

\section{EXPERIMENTAL PROCEDURE}

An ingot high in MnS content was made by melting $60 \mathrm{lbs}$ of 1119 steel under an argon atmosphere. Additions of $\mathrm{FeS}(0.25 \mathrm{lb})$ and electrolytic manganese $(0.425 \mathrm{lbs})$ were made at a superheat of $100^{\circ} \mathrm{C}$. These additions raised the manganese and sulfur contents to about $3.65 \%$ and $0.48 \%$ respectively. To obtain large inclusions, the melt was solidified in the crucible, simulating ingot solidification rates.

Three one-inch thick slices from the ingot were hot rolled at about $2000^{\circ} \mathrm{F}\left(1293^{\circ} \mathrm{C}\right)$ between 12-in. dia. rolls. Care was taken to ensure that the rolling direction was the same in each pass. The three slices were rolled to true thickness strains of $0.5,1.0$ and 1.5 , which correspond to reductions of $39 \%, 63 \%$ and $78 \%$. Image analyses indicated 


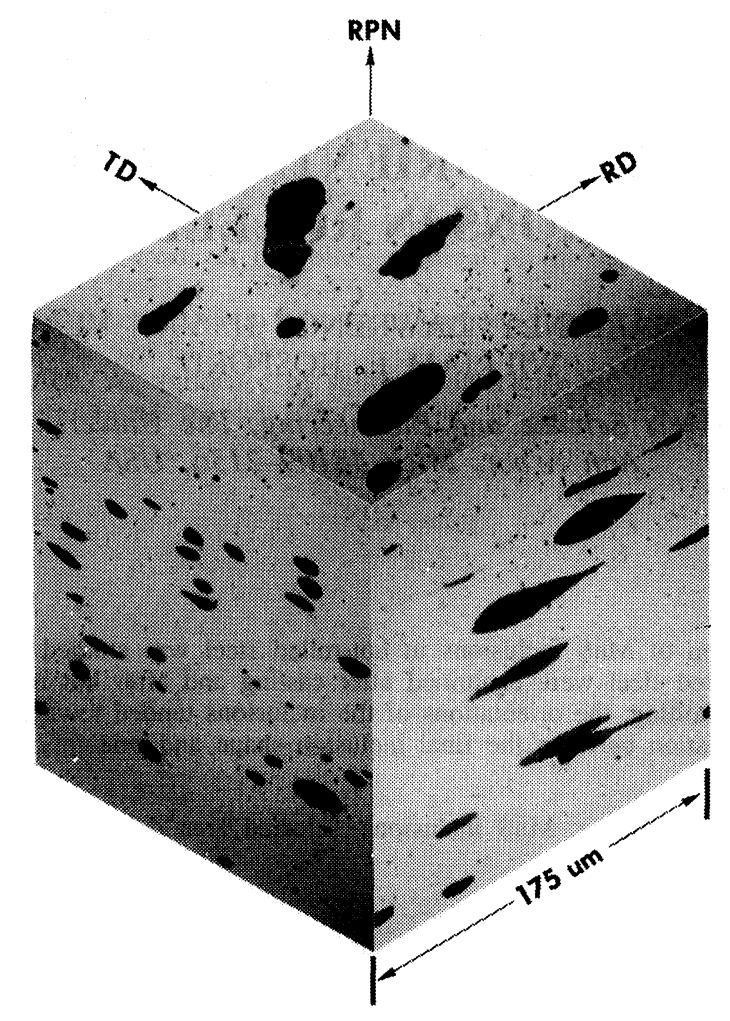

Figure 1 Manganese sulfide inclusions in hot-rolled steel after a rolling strain of 1.0.

that the strains of the inclusions were about two-third as the matrix strain. Figure 1 shows the microstructure after a rolling strain of 1.0.

Specimens for electron microscopy were obtained by cutting 1-mm thick slices perpendicular to the rolling direction. These were prepared for the transmission electron microscope by mechanical grinding followed by a final thinning in an argon ion mill. Because the inclusions were elongated in the direction perpendicular to the section, most of them extended through the thickness of the final sample.

Orientations of individual inclusions were determined using selected-area electron diffraction. Selected-area diffraction was chosen only after other methods including electron channeling, Kossel patterns, and electron back scattering from small polished areas were tried and found lacking, probably because of the high dislocation density. To avoid ambiguity, the sample was tilted until either a $\langle 100\rangle$ or $\langle 110\rangle$ direction was parallel to the beam. To ensure that the result were not biased by the selection of the inclusions to be examined, every inclusion with a sufficiently large electron-transparent area was studied. Many small inclusions could not be studied because they did not have large enough electron-transparent area. The orientation results for the inclusions studied were later checked to see whether there was a systematic orientation dependence on inclusion size. 

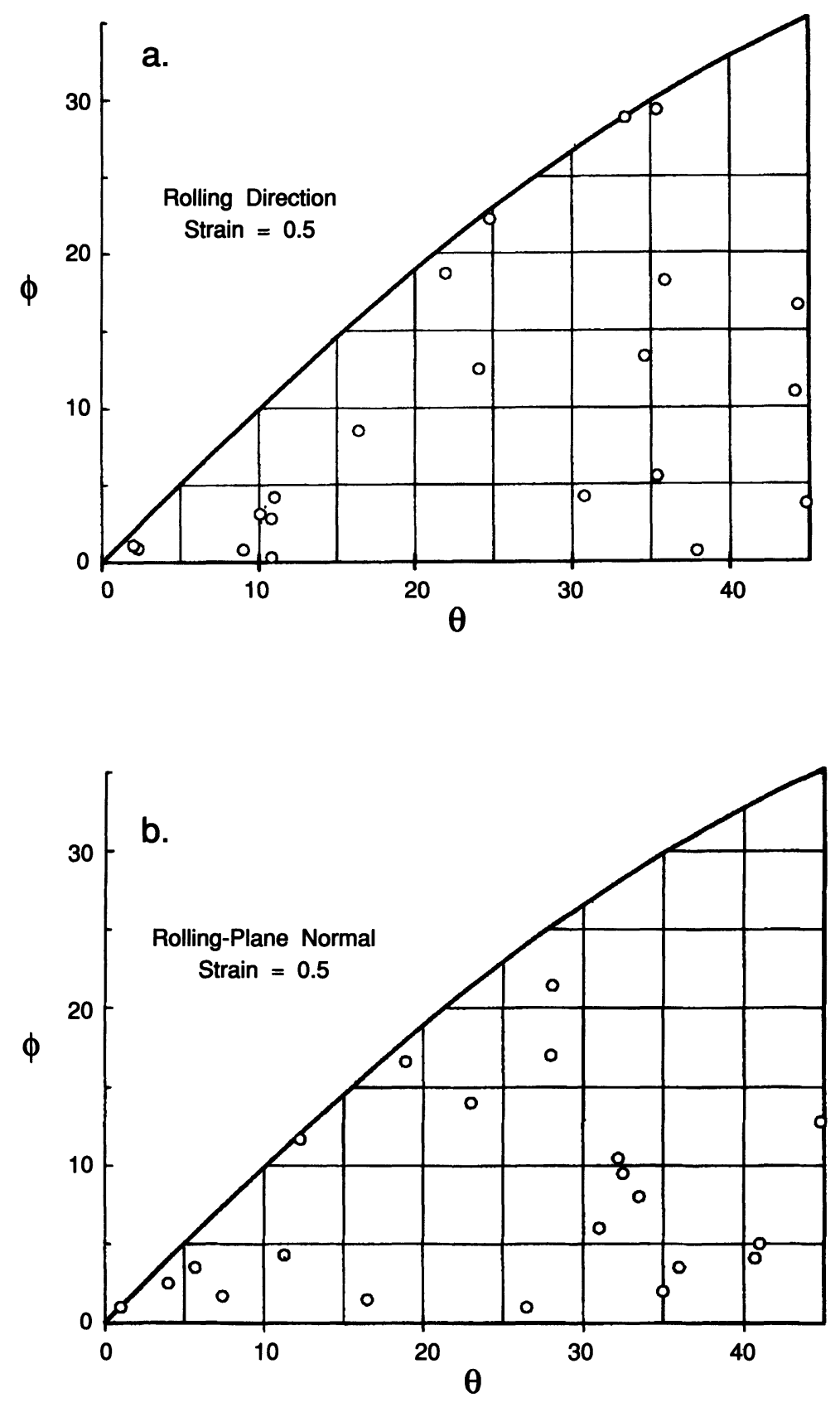

b

Figure 2 Inverse pole figures showing the orientations of the rolling direction (a) and rolling plane normal after a rolling strain of $\varepsilon=0.5$. Note that the orientations tend to cluster near $\langle 100\rangle$ and away from $\langle 111\rangle$. 

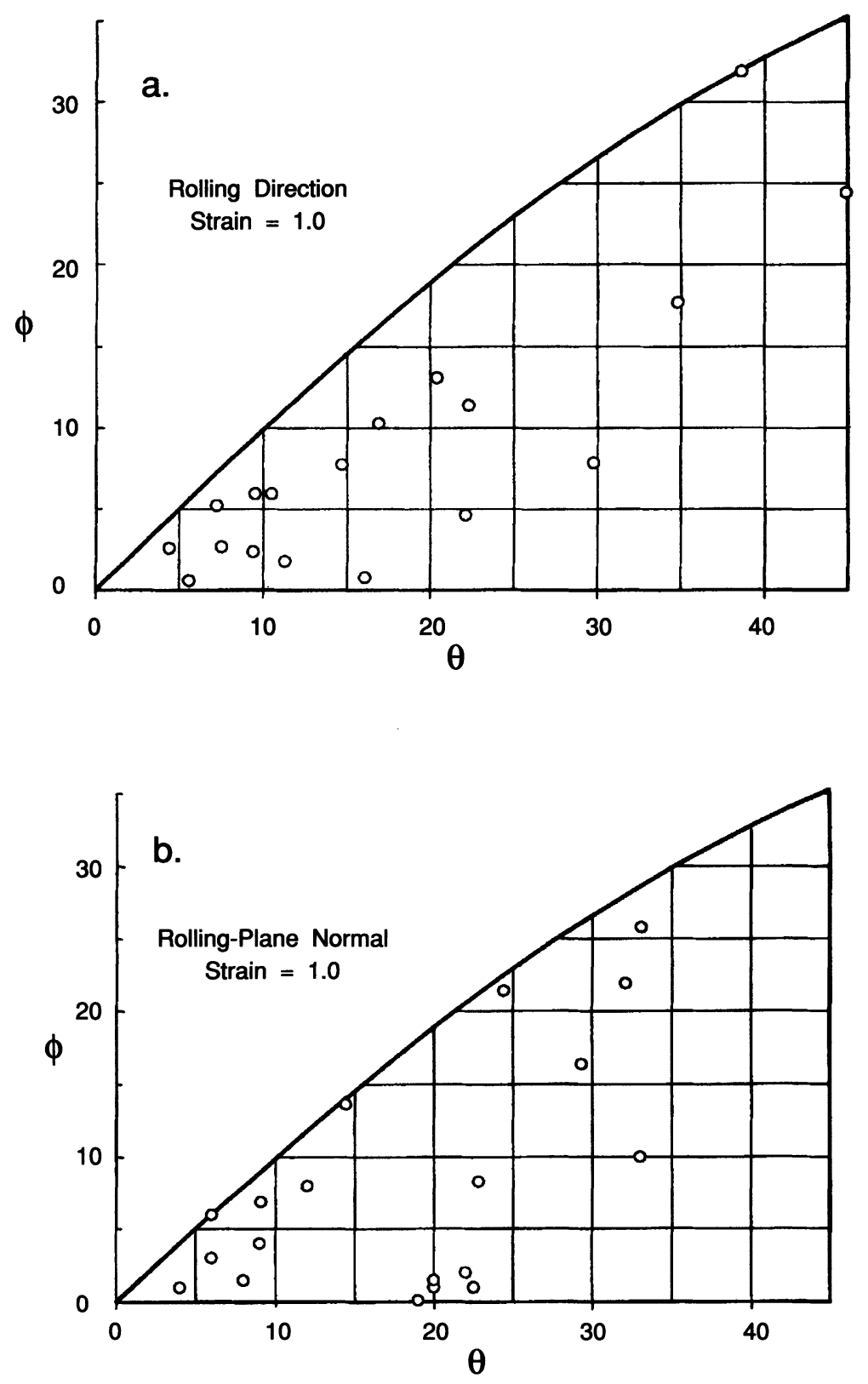

b

Figure 3 Inverse pole figures showing the orientations of the rolling direction (a) and rolling plane normal after a rolling strain of $\varepsilon=1.0$. Note that the orientations tend to cluster near $\langle 100\rangle$ and away from $\langle 111\rangle$. 

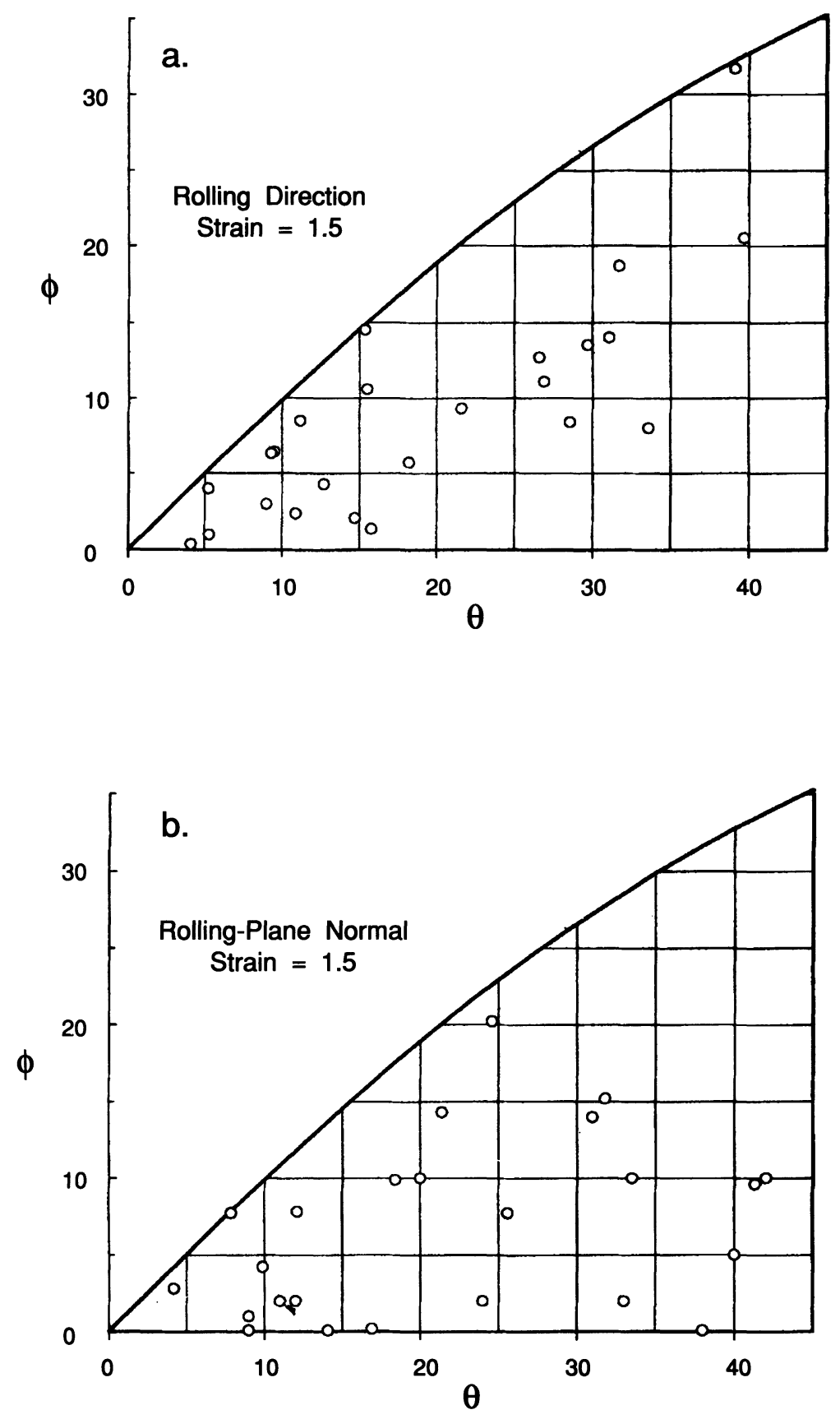

Figure 4 Inverse pole figures showing the orientations of the rolling direction (a) and rolling plane normal after a rolling strain of $\varepsilon=1.5$. Note that the orientations tend to cluster near $\langle 100\rangle$ and away from $\langle 111\rangle$. 


\section{PREFERRED ORIENTATION}

The orientations of the inclusions were plotted as inverse pole figures. For the as-cast material, the cylindrical axis of the ingot was chosen as a reference axis and no apparent clustering was observed. Inverse pole figures showing the orientations after rolling are given in Figures 2-4. Visual inspection of these figures suggests a tendency for the Rolling Direction (RD), Transverse Direction (TD) and Rolling-Plane Normal (RPN) to lie near a $\langle 100\rangle$ direction with some spread toward $\langle 110\rangle^{*}$. Absence of orientations near $\langle 111\rangle$ is apparent. However, with the limited number of inclusions studied, it is not possible to draw meaningful intensity contours.
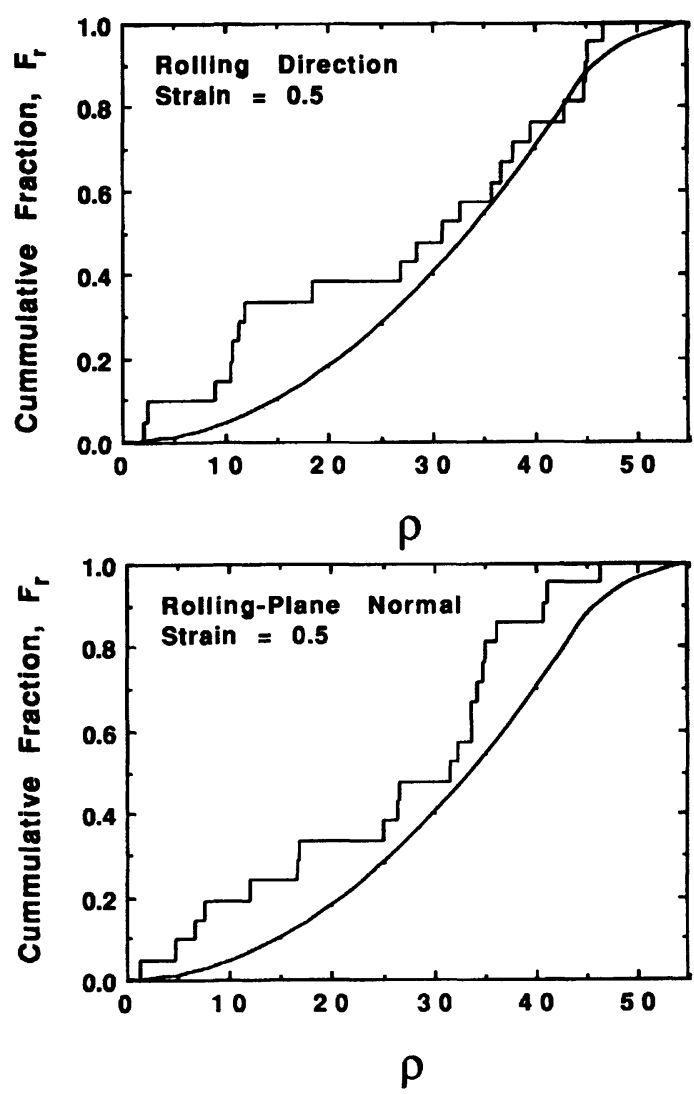

b

Figure 5 Cumulative fraction of inclusions oriented so that the rolling direction (a) and rolling plane normal (b) are oriented at an angle less than $\rho$ of an $\langle 001\rangle$ direction after a strain of $\varepsilon=0.5$. The experimental data (broken lines) are above what would be expected for a randomly oriented sample (continuous line) indicating a preferred orientation.

* The notation of RN, TD and RPN is equivalent to "longitudinal", "long transverse" and "short transverse" directions, respectively. 

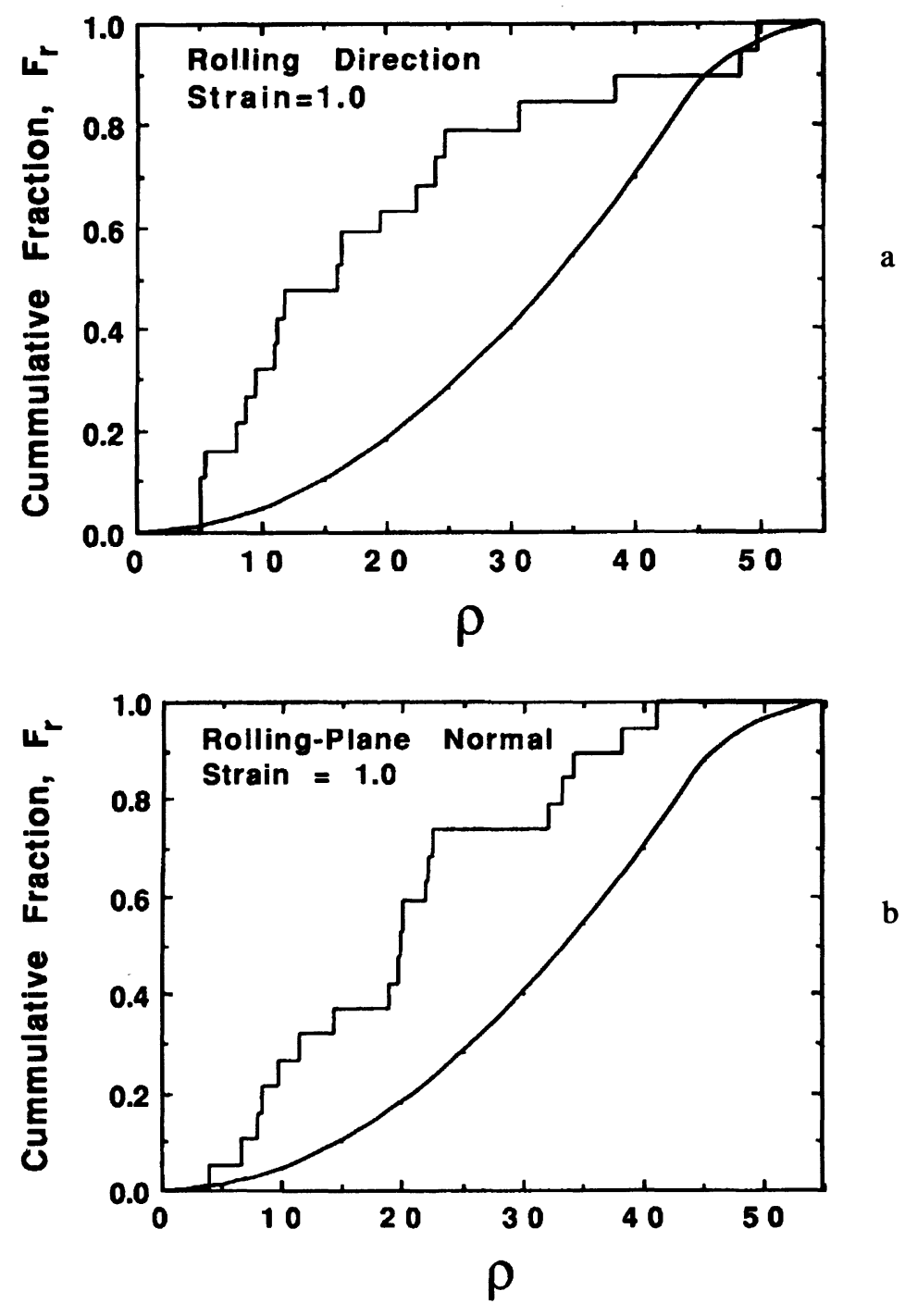

Figure 6 Cumulative fraction of inclusions oriented so that the rolling direction (a) and rolling plane normal (b) are oriented at an angle less than $\rho$ of an $\langle 001\rangle$ direction after a strain of $\varepsilon=1.0$. The experimental data (broken lines) are above what would be expected for a randomly oriented sample (continuous line) indicating a preferred orientation.

To assess the tendency toward $\langle 100\rangle$ ideal orientations, the cumulative fractions, $F$, of the orientations that lie at an angle, $\rho$, or less from the nearest $\langle 100\rangle$ axis were determined. Figures 5-7 show experimental plots F vs. $\rho$ as well as the distribution that would be expected for randomly oriented material. The values of $\rho$ were calculated from the orientation expressed by a "longitude," $\theta$, and a "latitude", $\phi$, using the relation

$$
\cos \rho=\cos \theta \cos \phi \text {. }
$$




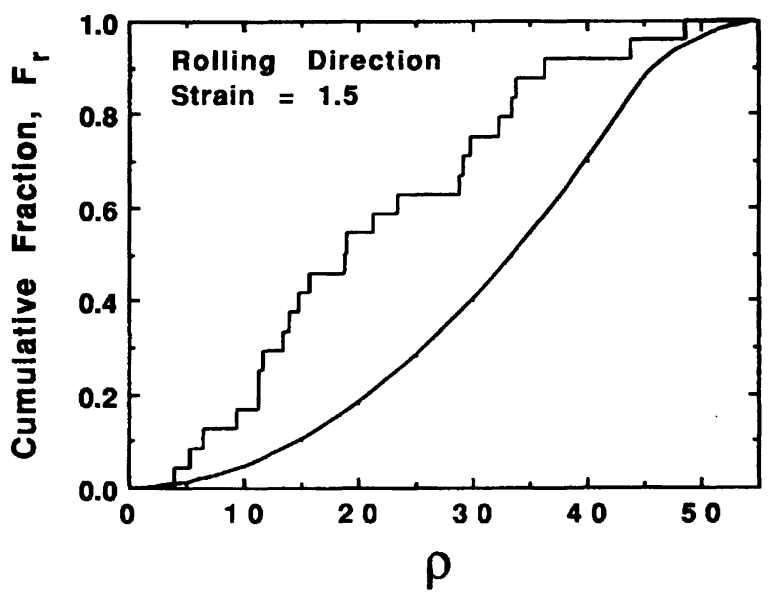

a

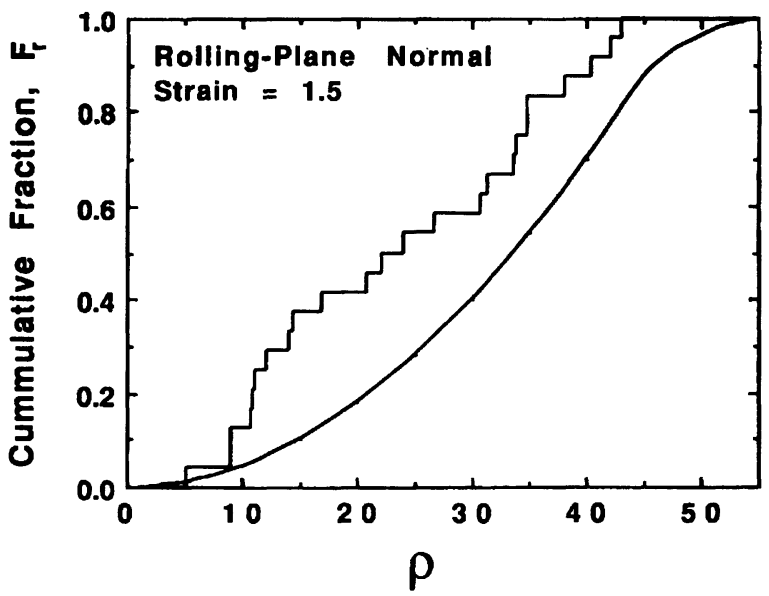

b

Figure 7 Cumulative fraction of inclusions oriented so that the rolling direction (a) and rolling plane normal (b) are oriented at an angle less than $\rho$ of an $\langle 001\rangle$ direction after a strain of $\varepsilon=1.5$. The experimental data (broken lines) are above what would be expected for a randomly oriented sample (continuous line) indicating a preferred orientation.

For $O \leq \rho \leq 45$, the fraction expected for randomly oriented material, $F_{r}$, can be calculated as

$$
\mathrm{F}_{\mathrm{r}}=3(1-\cos \rho) \text {. }
$$

For $45^{\circ} \leq \rho \leq 54.74^{\circ}, F_{r}$ was calculated numerically. At $\rho=54.74^{\circ}$, both $F$ and $\mathrm{F}_{\mathrm{r}}$ equal 1 .

A more quantitative assessment of data was made by plotting the normalized orientation density (NOD) vs. $\rho$. The normalized orientation density was calculated by dividing the fraction, $f$, of the inclusions oriented so that $\rho_{1}<\rho<\rho_{2}$ by the fraction of orientations that would be so oriented in random material. That is:

$$
\text { NOD }=f /\left[3\left(\cos \rho_{2}-\cos \rho_{1}\right)\right] \text {. }
$$


a.

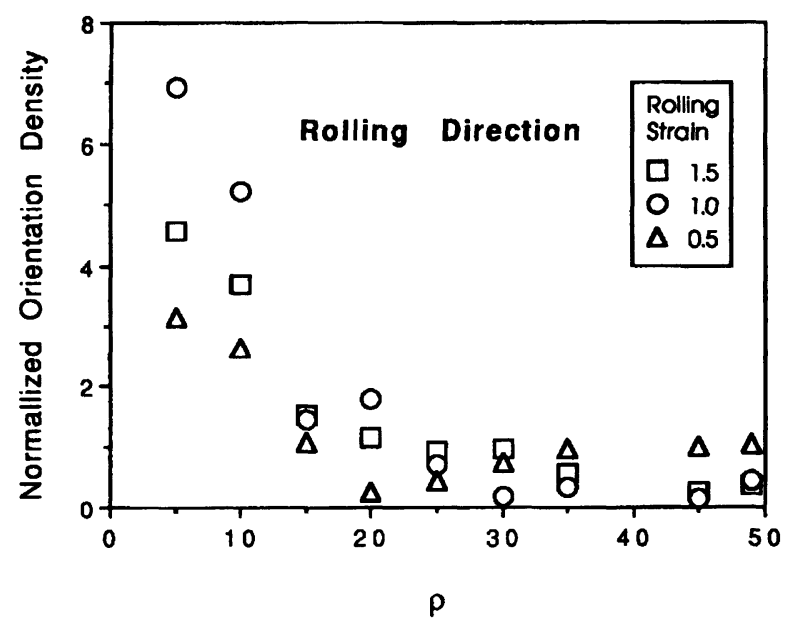

a

b.

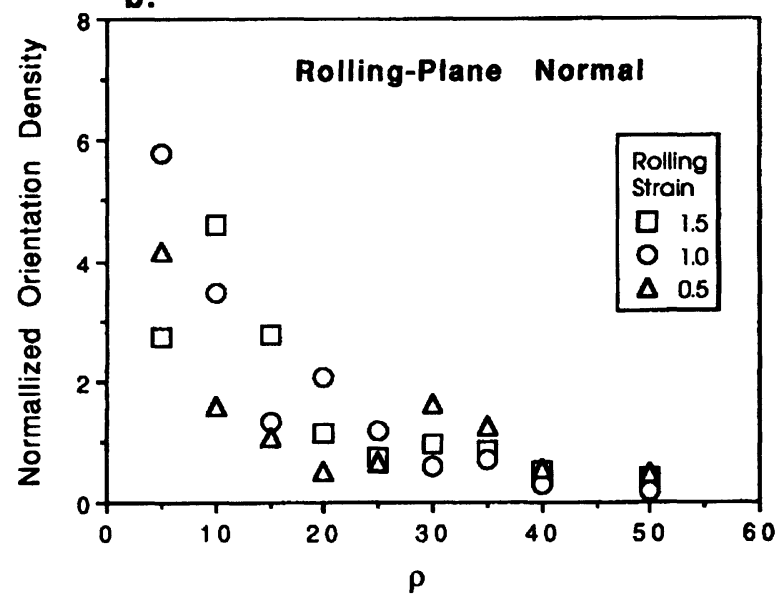

b

Figure 8 Normalized Orientation Density, NOD, as a function of $\rho$, for the rolling direction (a) and the rolling plane normal (b). The NOD is the density of orientations divided by the orientation density expected for a random sample. The figures indicate that NOD near $\langle 100\rangle$ increases with reduction up to $\varepsilon=1.0$.

Figures $8 a$ and $8 b$ are plots of NOD vs. $p$ for the RD and RPN after the various reductions. Because of the limited number of inclusions studied, the data were smoothed in constructing these plots, by taking $10^{\circ}$ increments of $\rho$ and plotting the NOD over this increment at the average $\rho$ in this increment. (i.e., the value of NOD plotted at $\rho=20^{\circ}$ represents the normalized density between $15^{\circ}$ and $25^{\circ}$.)

The intensity of the texture can be characterized by a single parameter, the Texture Index (TI), defined as the integral over the range $0^{\circ} \leq \rho \leq 54.7^{\circ}$ of $\mathrm{F}-\mathrm{F}_{\mathrm{r}}$ normalized by the maximum possible value of this integral.

$$
\mathrm{TI}=\int\left(\mathrm{F}-\mathrm{F}_{\mathrm{r}}\right) \mathrm{d} \rho / \int\left(1-\mathrm{F}_{\mathrm{r}}\right) \mathrm{d} \rho .
$$




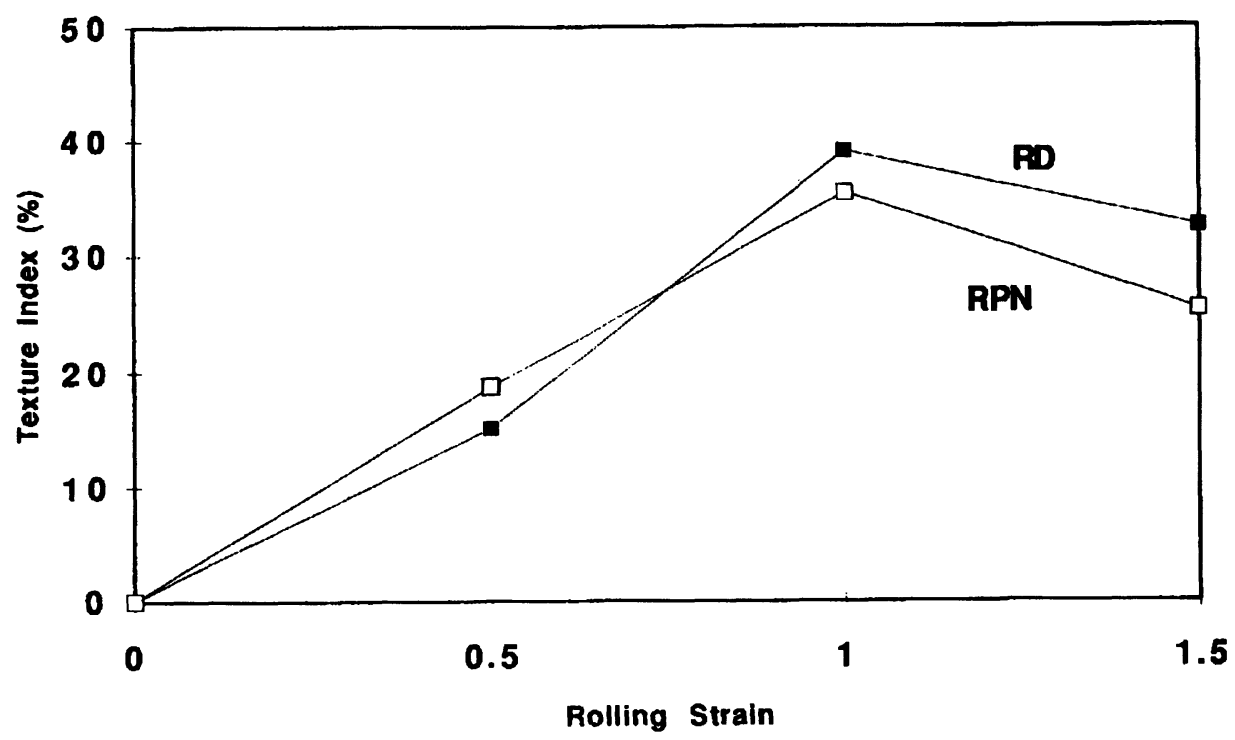

Figure 9 Increase of the strength of the texture with rolling strain. The texture index, TI, describes the texture intensity. For complete alignment, the TI would have a value of 1.0. The data shows that the strength of texture increases with reduction up to a strain of 1.0.

Figure 9 shows how the texture index changes with the rolling strain. This and the other figures all indicate the development of a $\langle 110\rangle\{110\}$ preferred orientation as the rolling increases from $\varepsilon=0.5$ to $\varepsilon=1.0$. Although analysis of the data for the starting cast material resulted in texture indexes of -8.7 and $12.2 \%$ for what would become the RD and RPN, Figure 9 was plotted with TI $=0$ for zero strain. Although Figures 8 and 9 indicate a stronger alignment of the rolling direction with $\langle 110\rangle$ at $\varepsilon=1.0$ than at $\varepsilon=1.5$, this observation probably reflects the limited number of observations rather than a real trend.

Great effort was made to randomly select inclusions for study. The only apparent bias of selection was one of inclusion size. The technique for determining the orientation requires that the inclusions be sufficiently large. The smaller inclusions were not studied. There is a possibility that this selection process could bias the data if there is a statistical correlation of orientation with inclusion size.

\section{DISCUSSION}

Manganese sulfide crystallizes in the $\mathrm{NaCl}$ structure. The predominant slip system is $\{110\}\langle 1 \overline{10} 0$. With $\{110\}\langle 1 \overline{1} 0\rangle$ slip, plane strain characteristic of flat rolling can be achieved most easily when the rolling direction is parallel to $\langle 100\rangle$ and the rolling plane if $\{001\}$.

One should expect that the relative deformation of the inclusions would depend on their orientation. For an isotropic material, the stress state for flat rolling is equivalent to tension in the rolling direction with compression normal to the rolling plane. The 
relative ease of deformation of any orientation may be characterized by a pseudo-Schmid factor.

$$
\mathrm{m}^{\prime}=\cos \lambda_{\mathrm{rd}} \cos \phi_{\mathrm{rd}}+\cos \lambda_{\mathrm{rpn}} \cos \phi_{\mathrm{rpn}}
$$

where $\lambda_{\mathrm{rd}}$ and $\phi_{\mathrm{rd}}$ are the angles between the rolling direction and the slip direction and slip plane normal respectively, and $\lambda_{\mathrm{rnn}}$ and $\phi_{\mathrm{rpn}}$ are the angles between the rolling plane normal and the slip direction and slip plane normal respectively. Figure 10 shows a weak correlation of the aspect ratio, $\mathrm{L} / \mathrm{W}$, of the inclusions with $\mathrm{m}$ ' after a rolling strain of 1.5 . For an inclusion that is initially spherical, the $\mathrm{L} / \mathrm{W}$ ratio is related to the thickness strain, $\varepsilon$ by

$$
\mathrm{L} / \mathrm{W}=\exp (2 \varepsilon)
$$

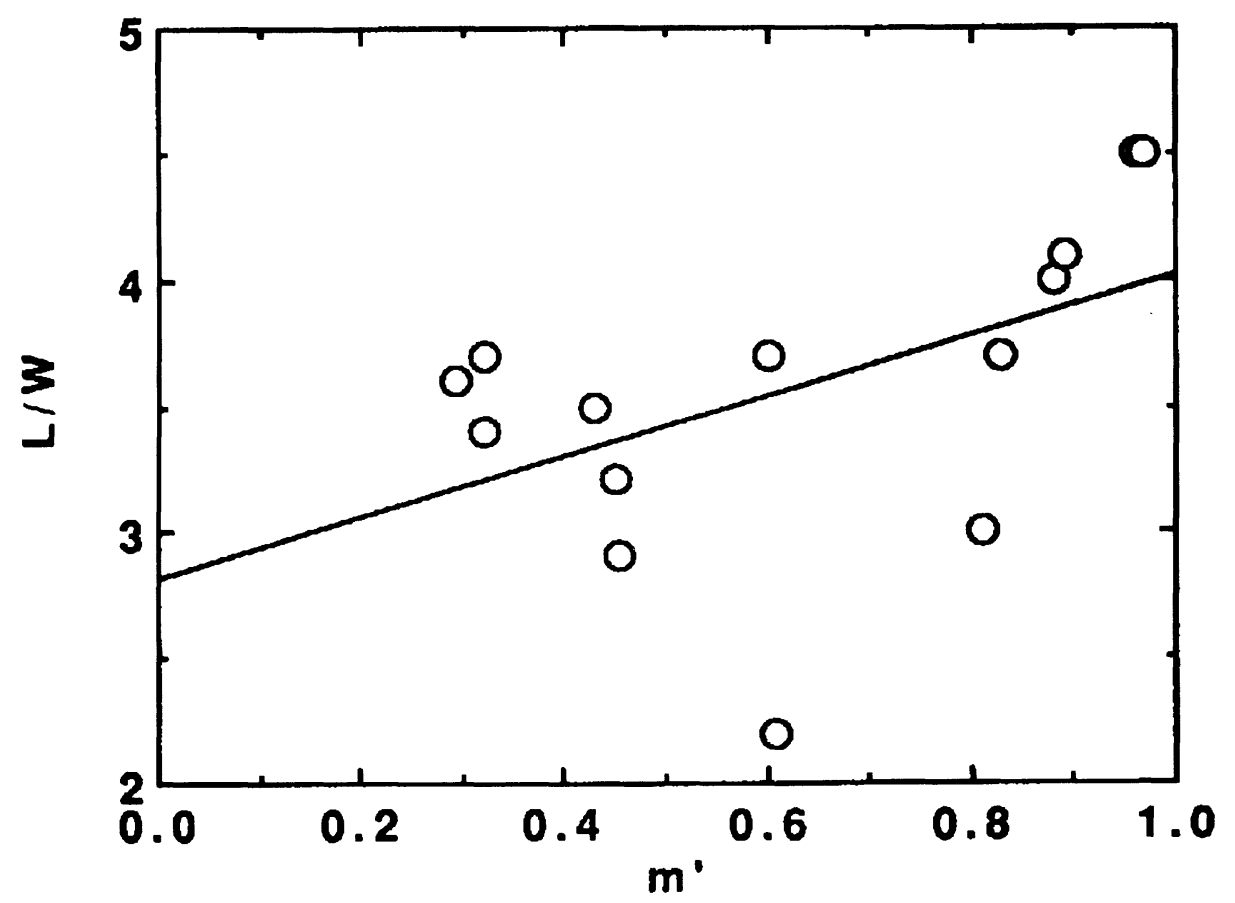

Figure 10 Correlation of the inclusion shapes after rolling to a strain of 1.5 with a pseudo-Schmid factor, $\mathrm{m}^{\prime}$, for the final orientation. The factor, $\mathrm{m}^{\prime}$ indicates the relative ease of slip. $\mathrm{L} / \mathrm{W}$ is the aspect ratio of the deformed inclusions.

so it can be concluded that favorably oriented inclusions tend to deform more than less favorably oriented inclusions.

The observed $\{100\}<001>$ preferred orientation gives the highest possible pseudoSchmid factor of 1.0. With this orientation the slip direction and the normal to the slip plane are both oriented at $\pm 45^{\circ}$ to both the rolling direction and the rolling-plane normal. 


\section{SUMMARY}

Manganese sulfide inclusions in steel have been found to have a $\{100\}<001>$ preferred orientation after hot rolling, The strength of the texture increases up to about a rolling strain of one.

\section{Acknowledgments}

The authors thank W. C. Bigelow for his help with some of the electron microscope work and Amax Laboratory for melting casting and hot rolling the material.

\section{References}

1. Chao, H. C., Thomassen L. and Van Vlack L. H., ASM Trans, Q., V. 57, pp. 386-98 (1964).

2. Chao H. C. and Van Vlack L. H., Trans. Met. Soc. AIME, v. 233, pp.1227-31 (1965).

3. Mardinly, A. J., "Crystallography and Microstructure of Manganese Sulfide Inclusions in Steel," $\mathrm{Ph}$. D. Thesis, University of Michigan (1982). 